\title{
Duty cycle and noise floor estimation with Welch FFT for Spectrum Usage Measurements
}

\author{
Kenta Umebayashi†, Ryota Takagi, \\ Naoki Ioroi, and Yasuo Suzuki \\ Tokyo University of Agriculture and Technology, \\ 2-24-16, Nakacho, Koganei-shi, Tokyo, Japan 184-8588 \\ Email: ume_k@cc.tuat.ac.jp $\dagger$
}

\author{
Janne J. Lehtomäki \\ Centre for Wireless Communications, University of Oulu \\ P. O. BOX 4500 FIN-90014 University of Oulu, Finland
}

\begin{abstract}
In dynamic spectrum access, a new wireless system (secondary user: SU) can utilize the spectrum licensed to an existing wireless system (primary user: PU) while the spectrum is vacant. For accomplishing effective and reliable spectrum utilization, statistics of the spectrum usage by the PU, such as duty cycle (DC), are useful for the SU. In this paper, we investigate accurate spectrum measurement technique with energy detector for obtaining the accurate duty cycle (DC) estimation with a noise floor (NF) estimation which is used for proper threshold setting. In one conventional method, median filtered FCME (Forward Consecutive Mean Excision), frequency selectivity due to interference among spectra of independent symbols degrades the NF and DC estimation performances. In this paper, we propose Welch FFT based NF estimation and DC estimation since Welch FFT has a potential to suppress the effect of the frequency selectivity. We analytically obtain probability density function (PDF) and right tail probability of output of the Welch FFT process and this analysis enables to set proper threshold. Numerical results will show that the proposed method can achieve better NF estimation performance. In addition, the NF estimation improvement and the suppression of frequency selectivity by the Welch FFT can provide around $15 \mathrm{~dB}$ SNR gain in the DC estimation performance.
\end{abstract}

\section{INTRODUCTION}

For the spectrum scarcity problem, dynamic spectrum access (DSA) with cognitive radio techniques by the unlicensed user (secondary user: SU) on the spectrum owned by the licensed user (primary user: PU) has been investigated [1]. In DSA, the SU can utilize the vacant spectrum as long as it will not cause any harmful interference to the PU. To find the vacant spectrum, spectrum sensing has been investigated [2].

The requirements for spectrum sensing are in fact very challenging. For example, detection probability has to be higher than $90 \%$ in significantly low signal to noise ratio (SNR $\gamma$ ) region, such as $-14 \mathrm{~dB}$ or less [3]. Therefore, achieving the requirement at low cost is difficult.

One of the possible approaches to achieve low cost and accurate spectrum sensing is use of the statistics PU spectrum utilization, such as duty cycle (DC), in spectrum sensing. Specifically, if DC of PU is available, SU can select stochastically vacant spectrum for spectrum sensing. Therefore, it is possible to achieve accurate spectrum sensing performance with low complexity [4], [5]. In addition, the statistics are useful for designing SU's medium access control protocol effectively [6]. Moreover, for more dynamic and flexible spectrum utilization in future wireless networks, spectrum utilization statistics will be very useful. In this approach, a development of accurate spectrum measurement system is necessary.

There are several spectrum measurement campaigns for accomplishing DSA [7], [8]. In most of the spectrum measurements, energy detector (ED) with threshold is used [9]. ED is very simple and does not require any prior information about the PU signal, however the detection performance strongly depends on the threshold setting [10], [11]. There are several criteria to set the threshold and the most appropriate approach is constant false alarm rate (CFAR). In this case, noise floor (NF) estimation is an important issue for the threshold setting, however most of the previous works have not considered this issue deeply but instead assumed the NF was known. In long term spectrum measurement, NF is not static [12] and therefore a periodic NF estimation is required.

Since in practice it can be difficult to get guaranteed noiseonly samples, one important issue in the NF estimation is that it is necessary to classify the observed samples into noise only samples denoted as clean samples, and noise and signal samples denoted as signal samples. FCME (Forward Consecutive Mean Excision) algorithm [13] has been proposed for the division process. However, the NF estimation performance is affected by outliers in the observed samples and biased estimation error.

An extended FCME algorithm, median filtered FCME with a correction factor $\beta$ (MED-FCME- $\beta$ ), has been proposed to resolve the issues [14], [15]. The median filter is used to avoid the effect of large outliers and it has been shown that MEDFCME- $\beta$ can achieve proper NF estimation performance. For DC estimation, localization algorithm based on doublethresholding with adjacent cluster combining (LAD-ACC) is used and it has also been shown that LAD-ACC based method can achieve proper DC estimation performance.

In this paper, we focus on a different issue, which is frequency selectivity, in NF and DC estimations. Similar problem has been observed in several works for example [16], [17]. Specifically, in the case that the actual DC is more than or equal to 0.5 and the signal to noise power ratio (SNR) is low such as $0 \mathrm{~dB}$, NF estimation performance with MED-FCME- $\beta$ may degrade. This degradation is due to frequency selectivity 


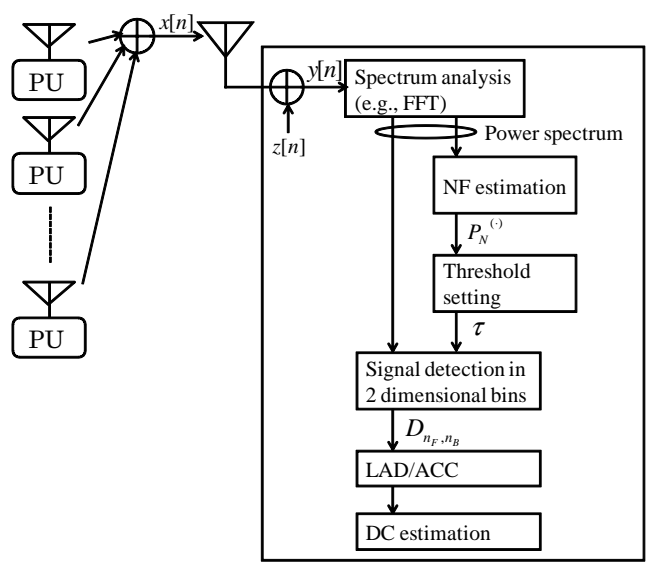

* Superscript $(\cdot)$ is an estimation method indicator.

Fig. 1: System model

caused by interference among spectra of independent symbols. In DC estimation, the frequency selectivity and NF estimation error degrade the DC estimation performance significantly. For this issue, we propose MED-FCME-Welch where the Welch FFT is employed [18], [19]. In the related works, [20], the Welch FFT has been used for spectrum measurement, especially for signal detection. On the other hand, in this paper, we mainly focus on the NF and DC estimations with Welch FFT. In the DC estimation process, LAD-ACC is also used. We will show that a significant gain in DC estimation performance can be provided by the proposed method and our approach can mitigate the effect of the frequency selectivity on the expense of frequency resolution.

\section{SySTEM MODEL}

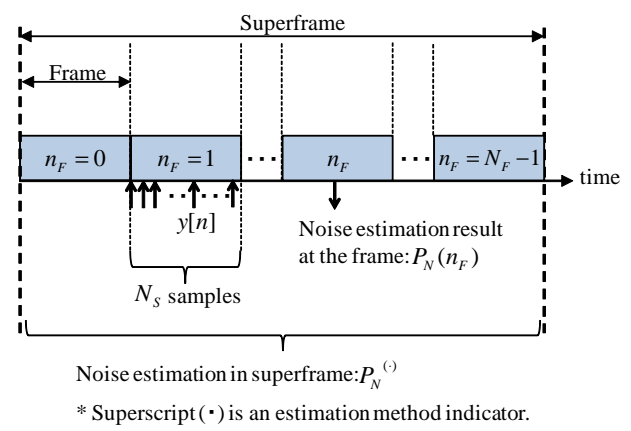

Fig. 2: Frame configuration and NF estimations

The system model is shown in Fig. 1. We assume that multiple PUs access the observed spectrum randomly. In addition, we assume that the observation equipment (OE) does not have any prior information about the spectrum occupancy by the PUs and PUs' signal specifications. This situation is similar to the spectrum utilization in the industry-sciencemedical (ISM) band where frequency bandwidth and time duration of occupancy are not constant and multiple spectrum occupancies can overlap. The purpose of the OE is to derive spectrum utilization detection results in two dimensional timefrequency grid composed of individual bins, i.e., vacant and occupied, denoted by $H_{0}$ and $H_{1}$, respectively.

The observation time frame is composed hierarchically and one superframe consists of $N_{F}$ frames as shown in Fig. 2. Now we focus on the $n_{F}$ th frame. During one frame, the OE obtains observed equivalent baseband signal $y[n](n=$ $\left.n_{F} N_{S}, n_{F} N_{S}+1, \cdots n_{F} N_{S}+N_{S}-1\right)$ with sampling rate $f_{s} \mathrm{~Hz}$ and the sampled complex signal is given by

$$
y[n]=x[n]+z[n]
$$

where $x[n]$ represents PU signal component, $z[n]$ represent noise component which is circularly symmetric normal complex random variable with zero mean and unit variance, in addition we assume flat NF in frequency domain. SNR is defined by $\gamma=E\left[|x[n]|^{2}\right] / E\left[|z[n]|^{2}\right]$ and $E[]$ is expectation operator. Without loss of generality $N_{S}$ is assumed to be power of two. The passband signal is located at the center frequency $f_{c}$ and the observed frequency $f$ is in the region where $f_{c}-f_{s} / 2 \leq f \leq f_{c}+f_{s} / 2$.

Power spectrum density for the given observed samples can be estimated with

$$
P_{n_{F}, n_{B}}=\left|\frac{1}{\sqrt{N_{S}}} \sum_{k=0}^{N_{S}-1}\left(y\left[k+n_{F} N_{S}\right] \times e^{-j 2 \pi n_{B} k}\right)\right|^{2}
$$

where $n_{F}$ is the index number for time frame, $n_{B}$ indicates frequency bin index number. The actual frequency $f\left(n_{B}\right)$ for given $n_{B}$ is given by

$$
f\left(n_{B}\right)= \begin{cases}\frac{n_{B} f_{s}}{N_{S}}+f_{c} & \left(0 \leq n_{B} \leq \frac{N_{S}}{2}+1\right) \\ \frac{\left(n_{B}-N_{S} / 2-1\right) f_{s}}{N_{S}}+f_{c} & \text { (otherwise), }\end{cases}
$$

where $n_{B}=0,1, \cdots, N_{S}-1$.

In the NF estimation block, NF estimation is performed every time frame and the estimated noise power is denoted by $P_{N}\left(n_{F}\right)$. In the conventional FCME algorithm, NF estimation $P_{N}\left(n_{F}\right)$ is used for the threshold setting. One of the possible approaches to enhance the NF estimation performance based on $N_{F}$ estimates is averaging. In fact, the errors caused by outliers or frequency selectivity provide biased estimation error in the NF estimation, therefore averaging is not effective as mentioned in [15].

On the other hand in the MED-FCME- $\beta$ and the proposed method, MED-FCME-Welch, the estimated NF is given by median filtering $N_{F}$ estimates in a superframe. The details of the NF estimation methods, FCME, MED-FCME- $\beta$, and MED-FCME-Welch, will be shown in the following sections.

In the threshold setting block, the threshold $\tau$ is set based on the target PFA $\dot{P}_{\mathrm{FA}}$. Specifically $\tau$ has to satisfy the following equation

$$
\dot{P}_{\mathrm{FA}}=\operatorname{Pr}\left(P_{n_{F}, n_{B}}>\tau \mid H_{0}\right),
$$

where $\operatorname{Pr}()$ indicates a probability for an event. 
In the signal detection block, based on the threshold $\tau$, signal occupancy is detected at two dimensional bin $\left(n_{F}, n_{B}\right)$ as

$$
D_{n_{F}, n_{B}}= \begin{cases}1 & \left(P_{n_{F}, n_{B}}>\tau\right) \\ 0 & \text { (otherwise) }\end{cases}
$$

Although median filter can provide accurate NF estimation, misdetection of the PU channel usage would still be possible with ED. For this issue, LAD-ACC is used in [15].

\section{NF ESTIMATION WITH MED-FCME- $\beta$}

MED-FCME- $\beta^{1}$ consists of FCME algorithm and median filter. For achieving accurate NF estimation, a compensation factor $\beta$ is also used.

\section{A. FCME algorithm}

FCME algorithm [21] is an iterative algorithm and $n_{i}$ denotes the index number of the iteration. Now we focus on the $n_{F}$ th frame and the $P_{n_{F}, n_{B}}$ sorted in ascending order are denoted by

$$
\left[\bar{P}_{n_{F}, 1} \bar{P}_{n_{F}, 2} \cdots \bar{P}_{n_{F}, c} \cdots \bar{P}_{n_{F}, N}\right],
$$

i.e., $\bar{P}_{n_{F}, n_{B}} \leq \bar{P}_{n_{F}, n_{B}+1}$.

The NF estimation in the first iteration is performed as follows. A set of clean samples is set by

$$
\mathbf{Q}(0)=\left\{\bar{P}_{n_{F}, 1}, \bar{P}_{n_{F}, 2}, \cdots, \bar{P}_{i, N_{Q}(0)}\right\}
$$

where $N_{Q}(0)=\left\lceil C N_{S} / 100\right\rceil$ and this indicates that at least $C \%$ samples are assumed to be clean samples.

An average of the clean samples in the 0th iteration $\mathbf{Q}(0)$ is given by

$$
\varsigma_{n_{F}}(0)=\frac{1}{N_{Q}(0)} \sum_{n_{B}=1}^{N_{Q}(0)} \bar{P}_{n_{F}, n_{B}}
$$

A threshold in the 0th iteration, $T_{h}(0)$, is given by

$$
T_{h}(0)=\varsigma_{n_{F}}(0) \cdot T_{\mathrm{CME}}
$$

where $T_{\mathrm{CME}}$ is set based on the target clean sample rejection rate (CSSR) denoted by $\dot{P}_{\mathrm{FA}, \mathrm{CSSR}}$ as

$$
T_{\mathrm{CME}}=-\ln \left(\dot{P}_{\mathrm{FA}, \mathrm{CSSR}}\right) \text {. }
$$

For $n_{i}>0, \mathbf{Q}\left(n_{i}\right)$ is updated as follows

$$
\mathbf{Q}\left(n_{i}\right)=\left\{\bar{P}_{n_{F}, n_{B}} \mid \bar{P}_{n_{F}, n_{B}}<T_{h}\left(n_{i}-1\right)\right\} .
$$

The number of the elements in $\mathbf{Q}\left(n_{i}\right)$ is also updated by $N_{Q}\left(n_{i}\right)=\left|\mathbf{Q}\left(n_{i}\right)\right|$. Moreover, $\varsigma_{n_{F}}\left(n_{i}\right)$ and $T_{h}\left(n_{i}\right)$ are updated as mentioned above.

The iterative process is continued while $N_{Q}\left(n_{i}\right)>N_{Q}\left(n_{i}-\right.$ 1 ) is satisfied and the final set of clean samples is denoted by $\mathbf{Q}\left(n_{\text {end }}\right)$ whhere $n_{\text {end }}=n_{i}-1$. This condition indicates that the iteration terminates if there are no new added elements in $\mathbf{Q}\left(n_{i}\right)$ at the $n_{i}$ th iteration. Then, the estimated NF in the $n_{F}$ th frame, $P_{N}\left(n_{F}\right)$, is given by $P_{N}\left(n_{F}\right)=\varsigma_{n_{F}}\left(n_{\text {end }}\right)$.

\footnotetext{
${ }^{1}$ In [15], the MED-FCME- $\beta$ is denoted by MED-FCME with a correction factor $\beta$.
}

\section{B. NF estimation with MED-FCME- $\beta$}

In the MED-FCME- $\beta$ [15], median filter is used for suppressing the effect of NF estimation error in one time frame $P_{N}\left(n_{F}\right)$. The NF estimation based on the median filter is shown as follows. $P_{N}\left(n_{F}\right)\left(n_{F}=0,1, \cdots, N_{F}\right)$ sorted in ascending order are given by

$$
\bar{P}_{N}(0) \leq \bar{P}_{N}(1) \leq \cdots \leq \bar{P}_{N}\left(N_{F}-1\right) .
$$

Then, the estimated NF by the median filter is given by

$$
P_{N}^{(\mathrm{MED})}= \begin{cases}\frac{\bar{P}_{N}\left(\frac{N_{F}}{2}\right)+\bar{P}_{N}\left(\frac{N_{F}}{2}+1\right)}{2} & \left(\text { even } N_{F}\right) \\ \bar{P}_{N}\left(\frac{N_{F}+1}{2}\right) & \left(\text { odd } N_{F}\right) .\end{cases}
$$

As mentioned in the previous subsection, $P_{N}\left(n_{F}\right)$ includes biased estimation error due to frequency selectivity. Averaging $P_{N}\left(n_{F}\right)$ can not effectively suppress the biased estimation error, therefore the median filter is used in [15]. However, the median filter is effective in the case where DC is less than $50 \%$ and if it is more than $50 \%$ it leads to some offset/bias.

In the FCME algorithm, it may involve biased NF estimation error due to the target CSSR. Specifically, $\dot{P}_{\mathrm{FA}, \mathrm{FCME}}$ of clean sampled can be eliminated by the FCME algorithm. To compensate the biased NF estimation error, the correction factor $\beta$ is used. The suitable correction factor $\beta$ is [15]

$$
\beta=10 \log 10\left(\sum_{j=1}^{N_{S}-\bar{h}} \frac{\left(N_{S}-\bar{h}-j+1\right)}{\left.\left(N_{S}-j+1\right)\left(N_{S}-\bar{h}\right)\right)}\right)
$$

where $\bar{h}$ is integer in the region $1 \leq \bar{h} \leq N_{S}$ and the smallest number satisfying

$$
\bar{h}=\left\lceil e^{T_{\mathrm{CME}}\left(\sum_{j=1}^{N_{S}-\bar{h}} \frac{\left(N_{S}-\bar{h}-j+1\right)}{\left.\left(N_{S}-j+1\right)\left(N_{S}-\bar{h}\right)\right)}\right)} N_{S}\right\rceil .
$$

The estimated NF in the MED-FCME- $\beta$ is given by $P_{N}^{(\mathrm{MED}-\beta)}=P_{N}^{(\mathrm{MED})}+\beta$.

For signal detection, the threshold $\tau$ is set by

$$
\tau=P_{N}^{(\mathrm{MED}-\beta)}+m,
$$

where $m$ is a shift value set to satisfy $\dot{P}_{\mathrm{FA}}$. Since $P_{n_{F}, n_{B}}$ under $H_{0}$ follows exponential distribution, the proper $m$ is given by

$$
m=10 \log 10\left(-\ln \left(\dot{P}_{\mathrm{FA}}\right)\right) .
$$

\section{DC ESTIMATION AND FREQUENCY SELECTIVITY}

\section{A. LAD-ACC method}

LAD-ACC algorithm is used for DC estimation. DC indicates signal occupancy rate in a certain frequency bin $^{2}$.

An example of the process of LAD-ACC is presented in Fig. 3. In the LAD, two thresholds, low threshold $\tau_{\mathrm{L}}$ and high threshold $\tau_{\mathrm{H}}$, are used and they are also set based on target CSSRs, $\dot{P}_{\mathrm{FA}, \mathrm{L}}$ and $\dot{P}_{\mathrm{FA}, \mathrm{H}}$, respectively. At first, in the step low (Fig. 3), signal is detected with low threshold. In

${ }^{2} \mathrm{DC}$ and channel occupancy rate are different parameters. In [15], DC and channel occupancy rate are defined. In this paper, we only focus on DC in a certain bin where PU signal exists randomly. 


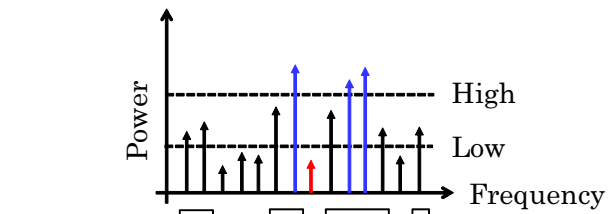

Step - Low 11000110111101

Step - High 00000110111100

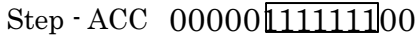

Fig. 3: Process of LAD-ACC.

fact, this detection result corresponds to $D_{n_{F}, n_{B}}$ in (5) with estimated PSD such as $P_{n_{F}, n_{B}}$. Contiguous detected samples are grouped together. In the example, there are four groups in the step low and each sample group is enclosed by a square.

The high threshold detects signal sample group where at least one sample in a sample group has to exceed the high threshold. In the example, there are two signal sample groups detected in the step high and they are enclosed by a square.

Finally, the ACC fills a gap between two detected groups if the gap is less than a predetermined number of samples. In this paper, we set the number of predetermined samples for filling to 3 .

The output of ACC is denoted by $A_{n_{F}, n_{B}}$ and DC for a bin $n_{B}$ is given by

$$
D C=\frac{1}{N_{F}} \sum_{k=0}^{N_{F}-1} A_{k, n_{B}}
$$

where $n_{B, \max }$ is the number of observed time samples in a superframe.

\section{B. Frequency selectivity}

In Figs, 4 and 5, transmit signal in time and frequency domains, respectively are shown. The upper two figures are in case of one symbol and the bottom two figures are in case of four symbols. Frequency spectrum in the case of one symbol (upper figure in Fig, 5) is almost rectangular since hamming window is used and in this case the region of signal can be detected by threshold $\tau$ easily. On the other hand, in the case of four symbols, the constructive and destructive interference among spectra of the four symbols provide the frequency selectivity (Fig. 5 bottom). The destructive interference causes NF estimation error since a sampled power spectrum in the signal bandwidth can be very close to NF. Although median filter and LAD-ACC have ability to suppress the effect of the frequency selectivity, it is not enough and the frequency selectivity in fact causes significant DC estimation error. This fact will be confirmed in numerical evaluations. In this paper, for this problem we propose a method using Welch FFT.

\section{NF estimation With MED-FCME-Welch FFT}

In the Welch FFT, $N_{S}$ samples are divided into $N_{\text {seg }}$ sample groups where each group consist of $N_{W}$ samples. In
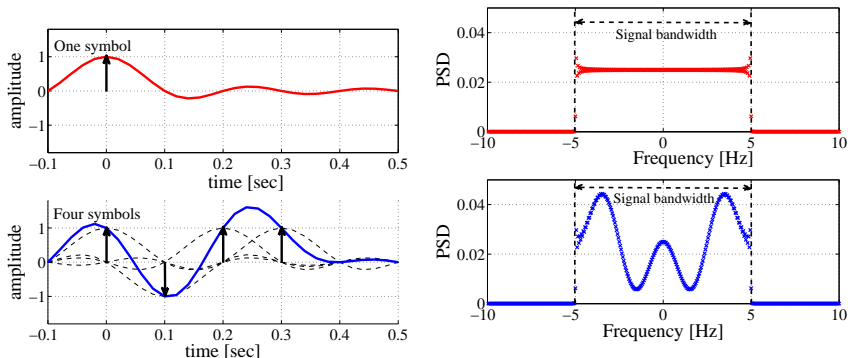

Fig. 4: Transmit signal in time domain. Upper one is one symbol case, Lower one is four symbols case.

Fig. 5: Transmit signal in frequency domain, Upper one is one symbol case, Lower one is four symbols case.

the $n_{\text {seg }}$ th segment, observed time complex samples $y_{n_{\text {seg }}}[n]$ $\left(n=0,1, \cdots, N_{W}-1\right)$ are given by

$$
y_{n_{\mathrm{seg}}}[n]=y\left[n+\left(n_{\mathrm{seg}}-1\right) N_{W} / 2\right],
$$

without loss of generality we assume $N_{W}$ is even number and there is overlapped samples between neighboring sample groups, i.e. the number of overlapped samples $N_{O}$ is set to $N_{W} / 2=N_{O}$. In this case, $N_{S}, N_{\text {seg }}$ and $N_{W}$ satisfies the following equation:

$$
N_{\text {seg }}=\frac{2 N_{S}}{N_{W}}-1
$$

where $N_{W}$ is assumed to be divisor of $2 N_{S}$.

Then, the discrete power spectrum density is given by

$$
P_{n_{\mathrm{seg}}, c_{\mathrm{seg}}}^{(\mathrm{seg})}=\left|\frac{1}{\sqrt{N_{W}}} \sum_{k=0}^{N_{W}-1}\left(h_{h}[k] y_{n_{\mathrm{seg}}}[k] e^{-j 2 \pi c_{\text {seg }} k}\right)\right|^{2}
$$

where $c_{\mathrm{seg}}$ is the index number of the frequency bin and $h_{h}[k]$ is hamming window given by

$$
h_{h}[k]= \begin{cases}0.54-0.46 \cos \left(\frac{2 \pi k}{N_{W}-1}\right) & \left(0<k<N_{W}-1\right) \\ 0 & \text { (otherwise) }\end{cases}
$$

In this case, the power spectrum density based on Welch FFT is given by

$$
P_{n_{F}, c_{s e g}}^{(\text {Welch })}=\frac{1}{N_{\text {seg }}} \sum_{n_{\text {seg }}=0}^{N_{\text {seg }}-1} P_{n_{\mathrm{seg}}, c_{\mathrm{seg}}}^{(\mathrm{seg}}
$$

Averaging process in the the above equation can suppress the frequency selectivity. Specifically, each spectrum in segment $P_{n_{\mathrm{seg},}, c_{\mathrm{seg}}}^{(\mathrm{seg}}$ has independent frequency selectivity characteristic and the averaging leads to nearly flat spectrum.

In the MED-FCME-Welch FFT, $P_{n_{F}, c_{s e g}}^{(\text {Welch })}$ under $H_{0}$ does not follow exponential distribution in contrast to $P_{n_{F}, n_{B}}$ but the $P_{n_{F}, c_{s e g}}^{\text {(Welch) }}$ is given by the sum of $P_{n_{F}, n_{B}}$. We assume that the effect of the overlap is negligible and $P_{n_{F}, c_{s e g}}^{(\text {Welch }}$ is denoted 


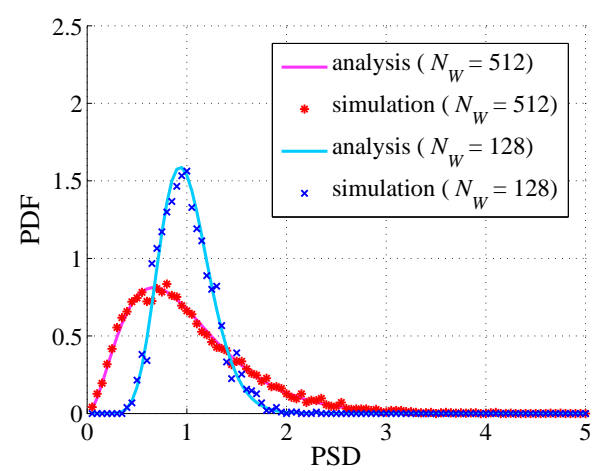

Fig. 6: PDF of $p(x)$ based on analysis (24) and Monte Carlo simulation result.

by $x$ for the sake of simplicity and $n_{x}=2 \times N_{\text {seg }}$ representing degrees of freedom. Then, the PDF of $x$ is given by [21]

$$
p(x)=\frac{n_{x}}{2^{\frac{n_{x}}{2}} \Gamma\left(\frac{n_{x}}{2}\right)}\left(n_{x} x\right)^{\left(\frac{n_{x}}{2}-1\right)} e^{\left(-\frac{n_{x} x}{2}\right)} .
$$

The FCME parameter $T_{\mathrm{CME}}$ for $\dot{P}_{\mathrm{FA}, \mathrm{CSSR}}$ has to satisfy

$$
\dot{P}_{\mathrm{FA}, \mathrm{CSSR}}=\int_{T_{\mathrm{CME}}}^{\infty} p(x) d x .
$$

In fact, the above equation can be expressed by an equivalent closed form equation as

$$
\begin{aligned}
& \dot{P}_{\mathrm{FA}, \mathrm{CSSR}}=\frac{n_{x}}{2^{\frac{n_{x}}{2}} \Gamma\left(\frac{n_{x}}{2}\right)} . \\
& \quad\left[\frac{\exp \left(\frac{n_{x} T_{\mathrm{CME}}}{2}\right)}{\frac{n_{x}}{2}} \sum_{r=0}^{\frac{n_{x}}{2}-1} \frac{(-1)^{r}\left(\frac{n_{x}}{2}-1\right) ! x^{\frac{n_{x}}{2}-1-r}}{\left(\frac{n_{x}}{2}-1-r\right) !\left(-\frac{n_{x}}{2}\right)^{r}}\right] .
\end{aligned}
$$

To confirm the validity of (24), the analysis and Monte Carlo simulation result are shown in Fig. 6.

The MED-FCME-Welch FFT can suppress the effect of frequency selectivity but the outliers still affect the NF estimation. Therefore, we employ the median filter and the estimation result is denoted by $P_{N}^{(\mathrm{MED}-\text { Welch })}$ and the threshold for signal detection is given by $\tau=P_{N}^{(\mathrm{MED}-\text { Welch })}+m$ where $m$ can be set based on the result in (26).

\section{NUMERICAL EVALUATIONS}

In this section, we evaluate NF and DC estimation performances in terms of MED-FCME- $\beta$ and MED-FCME-Welch FFT. Common simulation parameters in the computer simulation are as follows. Observed bandwidth is set to 2000 $\mathrm{Hz}$ and signal bandwidth is set to $440 \mathrm{~Hz}$ and noise power is set to unit power. There are three target probabilities, the first one is CSSR for setting $\dot{P}_{\mathrm{FA}, \mathrm{CSSR}}$ for setting $T_{\mathrm{CME}}$, the second one is $\dot{P}_{\mathrm{FA}, \mathrm{L}}$ for setting $\tau_{L}$, and the third one is $\dot{P}_{\mathrm{FA}, \mathrm{H}}$ for setting $\tau_{H}$. They are set as follows; $\dot{P}_{\mathrm{FA}, \mathrm{CSSR}}=0.01$, $\dot{P}_{\mathrm{FA}, \mathrm{H}}=4.54 \cdot 10^{-5}$, and $\dot{P}_{\mathrm{FA}, \mathrm{L}}=0.01$. These PFA settings are same setting as in [15]. FFT size $N_{S}$ and segment size in Welch FFT $N_{W}$ are set to 1024 and 64, respectively. In fact, $N_{W}$ setting is an important issue, but as space is limited, we are not concerned with this issue.

\section{A. NF estimation performance}

Fig. 7 shows the average estimated NF in decibel as a function of SNR for different DC (0.1, 0.5 and 0.9). The estimated NF is normalized by the preset actual noise power, then the true value is $0 \mathrm{~dB}$ in Fig. 7.

In the case of high SNR $(\gamma=20 \mathrm{~dB})$, both methods (MEDFCME- $\beta$ and MED-FCME-Welch FFT) achieve accurate NF estimation for any DCs. On the other hand, in the case of low SNR $(\gamma=0 \mathrm{~dB})$, the estimation error in the MEDFCME- $\beta$ increases as the DC increases. This indicates that median filter is effective only in the low DC case. Specifically, in low DC such as $0.1,90 \%$ of observed spectra are only noise component thus accurate NF estimation is possible and median filter can remove the effect of frequency selectivity. On the other hand, in case that DC is more than or equal to 0.5 , median filter can not always remove the effect of frequency selectivity. However, the MED-FCME-Welch FFT can suppresses the effect of frequency selectivity directly and it is possible to achieve accurate NF estimation even in the low SNR case.

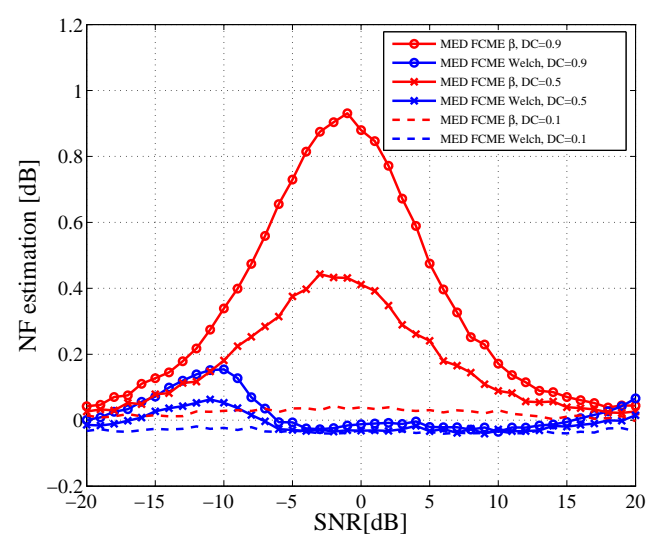

Fig. 7: NF estimation as a function of SNR. $\mathrm{DC}=0.1 .0 .5$ and 0.9. MED-FCME- $\beta$ and MED-FCME-Welch FFT.

\section{B. DC estimation performance with real measurement results}

We also evaluate the DC estimation performances with MED-FCME- $\beta$ and MED-FCME-Welch FFT in Fig. 8. The real DCs are set to $0.1,0.5$ and 0.9 In this evaluation, DC estimation is obtained by two cases: in the first case NF estimation methods (MED-FCME- $\beta$ and MED-FCME-Welch FFT) are used and in the second case perfect NF is available. The first case is plotted by solid line and the second case is plotted by dot line.

As confirmed in Fig. 7, MED-FCME-Welch FFT can achieve accurate NF estimation therefore, the gap in DC estimation between two cases in MED-FCME-Welch FFT is small. On the other hand, there is around one $\mathrm{dB}$ SNR difference between two cases in MED-FCME- $\beta$ when DC is 0.5 and 0.9. This result indicates that the difference comes from NF estimation error. 
The DC estimation difference between MED-FCME- $\beta$ and MED-FCME-Welch FFT is around $15 \mathrm{~dB}$. This significant difference is due to Welch FFT.

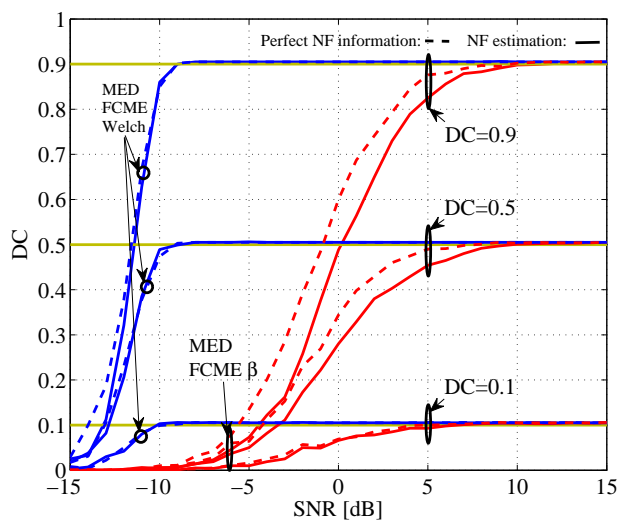

Fig. 8: DC estimation as a function of SNR. $\mathrm{DC}=0.1$. 0.5 and 0.9. MED-FCME- $\beta$ and MED-FCME-Welch FFT.

\section{CONCLUSION}

In this paper, we have investigated a spectrum measurement technique where NF estimation, spectrum usage detection in a two-dimensional time-frequency grid, and DC estimation are performed. For spectrum usage detection, ED was used and therefore threshold setting based on periodical NF estimation is important for long time spectrum measurement. Frequency selectivity degrades NF and DC estimation performances in the conventional method (MED-FCME- $\beta$ ). In our proposed NF estimation, Welch FFT is used and it can suppress the effect of the frequency selectivity on the expense of frequency resolution. For the threshold setting, we analyzed the PDF and right tail probability for observed periodogram by the Welch FFT. The numerical results verified the efficiency of the proposed method. Specifically, accurate NF estimation was available in low SNR case such as $\gamma=0 \mathrm{~dB}$ even if DC is more than 0.5. In addition, in terms of DC estimation, our proposed method can achieve around $15 \mathrm{~dB}$ gain compared to MED-FCME- $\beta$.

\section{ACKNOWLEDGMENT}

This research was supported by the Strategic Information and Communications R\&D Promotion Programme (SCOPE).

\section{REFERENCES}

[1] I. F. Akyildiz, W. Y. Lee, M. C. Vuran, and S. Mohanty, "Next generation/dynamic spectrum access/cognitive radio wireless networks: A survey," Computer Networks: The International Journal of Computer and Telecommunications Networking, vol. 50, pp. 2127-2159, Sept. 2006.

[2] T. Yucek and H. Arslan, "A survey of spectrum sensing algorithms for cognitive radio applications," IEEE Communications Surveys \& Tutorials, vol. 11, pp. 116-130, 2009.

[3] FCC, "Second report and order and memorandum opinion and order: in the matter of unlicensed operation in the TV broadcast bands," FCC, Tech. Rep. Doc. 08-260, Nov. 2008.
[4] D. Zhao and X. Zhou, "Spectrum sensing using prior probability prediction," in in Proc. of Wireless Communications, Networking and Mobile Computing (WiCOM), Sep. 2011, pp. 1-4.

[5] J. Vartiainen, M. Höyhtyä, J. Lehtomaki, and T. Braysy, "Priority channel selection based on detection history database," in in Proc. of IEEE Cognitive Radio Oriented Wireless Networks \& Communications (CROWNCOM), June 2010, pp. 1-5.

[6] K. Umebayashi, Y. Suzuki, and J. Lehtomäki, "Dynamic selection of CWmin in cognitive radio networks for protecting IEEE 802.11 primary users," in Proc. CROWNCOM, June 2011, pp. 266-270.

[7] M. Fitch, M. Nekovee, S. Kawade, K. Briggs, and R. MacKenzie, "Wireless service provision in TV white space with cognitive radio technology: A telecom operator's perspective and experience," IEEE Communications Magazine, vol. 49, no. 3, pp. 64-73, 2011.

[8] T. M. Taher, R. B. Bacchus, K. J. Zdunek, and D. A. Roberson, "Longterm spectral occupancy findings in Chicago," in in Proc. of IEEE Symposium on Dynamic Spectrum Access Networks, May 2011, pp. 100107.

[9] H. Urkowitz, "Energy detection of unknown deterministic signals," Proc. IEEE, vol. 55, no. 4, pp. 523-531, Apr. 1967.

[10] A. Sahai, N. Hoven, and R. Tandra, "Some fundamental limits on cognitive radio," in in Forty-second Allerton Conference on Communication, Control, and Computing, 2004

[11] K. Patil, R. Prasad, and K. Skouby, "A survey of worldwide spectrum occupancy measurement campaigns for cognitive radio," in in Proc. of Devices and Communications (ICDeCom), Feb. 2011, pp. 1-5.

[12] D. Torrieri, "The radiometer and its practical implementation," in in Proc. of IEEE MILCOM, Oct./Nov. 2010.

[13] H. Saarnisaari and P. Henttu, "Impulse detection and rejection methods for radio systems," in in Proc. of IEEE Military Communications Conference, Oct. 2003, pp. 1126-1131.

[14] J. J. Lehtomäki, R. Vuohtoniemi, K. Umebayashi, and J.-P. Mäkelä, "Energy detection based estimation of channel occupancy rate with adaptive noise estimation," IEICE Trans. Commun., vol. E95-B, no. 4, pp. 1076-1084, Apr. 2012.

[15] J. J. Lehtomaki, R. Vuohtoniemi, and K. Umebayashi, "On the measurement of duty cycle and channel occupancy rate," IEEE J. Select. Areas Commun., vol. 31, no. 1, pp. 2555 - 2565, Nov. 2013.

[16] J. J. Lehtomäki, J. Vartiainen, M. Juntti, and H. Saarnisaari, "Spectrum sensing with forward methods," in Proc. IEEE Military Communications Conference (MILCOM), Washington DC, Oct. 2006, pp. 23-25.

[17] J. Lehtomaki, M. Juntti, and H. Saarnisaari, "CFAR strategies for channelized radiometer," IEEE Signal Processing Lett., vol. 12, pp. 1316, Jan. 2005

[18] P. D. Welch, "The use of fast fourier transform for the estimation of power spectra: A method based on time averaging over short, modified periodograms," IEEE Trans. Audio Electroacoust., vol. 15, pp. 70-73, June 1967.

[19] H. Sarvanko, M. Mustonen, A. Hekkala, A. Mammela, M. Matinmikko, and M. Katz, "Cooperative and noncooperative spectrum sensing techniques using welch's periodogram in cognitive radios," in in Proc. of Cognitive Radio and Advanced Spectrum Management international workshop, Feb. 2008, pp. 1-5.

[20] J. Lehtomaki, S. Salmenkaita, J. Vartiainen, J.-P. Makela, R. Vuohtoniemi, and M. Juntti, "Measurement studies of a spectrum sensing algorithm based on double thresholding," in in Proc. of Cognitive Radio and Advanced Spectrum Management international workshop, May 2009, pp. 69-73.

[21] H. Saarnisaari, P. Henttu, and M. Juntti, "Iterative multidimensional impulse detectors for communications based on the classical diagnostic methods," IEEE Commun. Lett., vol. 53, pp. 395-398, Mar. 2005. 